\title{
The heart and the brain quantic interaction
}

\author{
Mordeniz Cengiz* \\ Department of Anesthesiology, Intensive Care, and Pain Medicine, Medical Faculty of Namik Kemal University, Turkey
}

\section{Communication}

Quantum coherence involves a precise phase-correlated coupling between body rhytms such as the constant communication that occurs between the human heart and the rest of the body. Increased coherence in a single system, such as in the electromagnetic field of the heart, can drive coordination of related physiologic systems within the body. Research studying heart rate variability revealed that an individual's emotional states can be directly correlated to cardiac electromagnetic coherence. Positive coping mechanisms and intention have been shown to increase cardiac coherence, whereas negative emotions result in diminished coherence.

As the most powerful and consistent generator of rhythmic information patterns in the body, the heart is in continuous communication with the brain and whole body through multiple pathways: neurologically, (through the Autonomic Nervous System (ANS)) biochemically (through hormones), biophysically (through pressure and sound waves), and energetically (through electromagnetic field interactions). Because of the extensiveness of the heart's influence on physiological, cognitive, and emotional systems, the heart provides a central point of reference from which the dynamics of such processes can be regulated.

The heart is particularly sensitive and responsive to changes in a number of other psychophysiological systems, especially changes in the activity of either branch of ANS. The heart's intrinsic network of sensory neurons detect and respond to variations in hormonal levels in the blood flow and efferent ANS signals. In addition to functioning as a sophisticated information processing and encoding center, the heart is also an endocrine gland that produces and secretes hormones and neurotransmitters. The complex circuitry of the heart senses, remembers, selfregulates, and makes decisions about cardiac control independent of the central nervous system.

The heart's sensory neurons translate hormonal and mechanical information into neurological impulses which are processed in the intrinsic nervous system and then sent to the brain via afferent pathways in the vagus nerve and spinal cord. Given the central role of the heart in creating coherence and positive emotions, it is not surprising that one of the strongest common threads uniting the views of diverse cultures, religious and spiritual traditions throughout human history, has been the universally the human heart as the source of love, wisdom, intuition and positive emotions. The heart's energy field (energetic heart) is coupled with a field of information that is not bound by the classical limits of time and space. This evidence comes from a rigorous experimental study showing that the body receives and processes information about a future event before the event actually happens. The heart receives intuitive information before the brain, and sends a different pattern of afferent signals. In this way, the heart is directly coupled with a subtle energetic field of entangled information that interacts with multiple energetic fields in which the body is embedded - including the quantum vacuum.

Within each individual, the level of stress and degree of global and heart coherence fluctuate from day to day, as does the quality of our thoughts and emotions. The cardiovascular system modulates cortical functions via afferent input from the baroreceptors in the heart, aortic arch, and carotid arteries. The heart's activity attunes cognitive performance, which actually fluctuates across the entire cardiac cycle at a rhythm around $10 \mathrm{~Hz}$. The influence on cortical function is mediated via a synchronizing effect on the neurons in the thalamus, which in turn synchronizes global cortical activity. It is the pattern (the rhythm) of the afferent input within the cardiac cycle, rather than the number of neural bursts.

Interventions providing emotional self-regulation skills combined with heart rhythm coherence training, result in improved workplace communication, satisfaction, productivity, innovative problem solving and reduced employee turnover. When an individual is in heart coherence, the heart radiates a more coherent electromagnetic signal into the environment, which can be perceived by nearby animals or the nervous systems of other people. Of all the organs, the heart generates the largest rhythmic electromagnetic field (EM), approximately 5,000 times stronger than the brain's EM. This magnetic field, which can be detected with sensitive magnetometers from the body, is a mechanism for how we can "feel" or sense another person's presence and emotional state, independent of body language, or other factors. By reinforcing the natural coupling in the sub-cortical regulatory systems, the selfactivation of a positive feeling automatically initiates an increase in cardiac coherence, while a physiological shift resulting from heartfocused breathing facilitates the experience of a positive emotion, related to the level of coherence of the heart rhythm pattern.

It is clear that responding in healthy and effective ways to ongoing inner and outer demands and circumstances, such as daily life situations, depends to a great extent on the synchronization, sensitivity, and stability of our physiological systems. If the neural systems are destabilized by trauma, stress, anxiety or chemical stimulants, the baseline reference patterns become unstable and unsettled emotions, and atypical reactions can be experienced.

In addition to afferent nerve activity associated with mechanical information such as pressure and rate that occurs at each heartbeat, continuous dynamically changing patterns of afferent activity related to chemical information is sent to the brain and other systems in the

${ }^{\star}$ Correspondence to: Mordeniz Cengiz, Department of Anesthesiology, Intensive Care, and Pain Medicine, Medical Faculty of Namik Kemal University, Tekirdag, Turkey, Tel: +905055107675; Fax: 90282250 9950; E-mail: cengizmorster@gmail.com

Received: April 22, 2020; Accepted: May 12, 2020; Published: May 14, 2020 
body. In terms of emotional experience, there are afferent pathways which synchronize the activity in the central nucleus of the amygdala via the Nucleus of Tractus, with the cardiac cycle.Therefore, the afferent inputs from the cardiovascular system to the amygdala are important contributors in determining emotional experience and in establishing the set point to which the current inputs are compared.

In most social contexts, individuals at times experience incoherent feelings towards one another, such as preconceptions or judgments, which are unspoken and can result in disruptions in optimal social interactions through miscommunication or other damaging social dynamics. In addition to generating unpleasant feelings and relational dynamics, engaged physiological processes have a direct bearing on our state of health. Individuals learn to sustain coherence while communicating by increased physiological linkage, and become more sensitive to others to promote greater empathy and rapport, for the process of heart felt connection to occur. For example, individuals in incoherent social situations, including social chaos or isolation, are more susceptible to disease.

The physiological activity associated with sustained positive emotions enhances synchronization of neurological activity and improves the cognitive functions that generate rational thought, creativity, and intentional action. The positive emotions give rise to a distinct mode of physiological functioning, termed psychophysiological coherence. Physiological correlates of this mode include a smooth, sine wave-like pattern in the heart rate variability trace (heart rhythm coherence), which reflects increased synchronization between the activity in the two branches of the autonomic nervous system; a shift toward increased parasympathetic activity; increased heart-brain synchronization (the brain's rhythms become more synchronized to the heartbeat); increased vascular resonance; and entrainment among diverse physiological oscillatory systems. These individual's physiological and psychological processes changes result in a highly efficient state in which the body, brain, and nervous system function with increased order, synchronization and harmony.Increasing psychophysiological coherence is associated with improvements in cognitive performance on tasks requiring focus and attention, discrimination, quick and accurate reaction, and memory. Moreover, the coherence mode is also associated with increased emotional stability and a reduction in the perception of stress and negative emotions. At the physiological level, this mode manifests as more ordered activity and synchronous interactions among the body's systems, encompassing biophysical phenomena such as autocoherence, entrainment, synchronization, and resonance.

During the psychophysiological coherence mode, there is increased cross-coherence or entrainment among the rhythmic patterns of activity generated by different physiological oscillatory systems. The heart is the body's most powerful rhythmic oscillator and generates the strongest rhythmic wave pattern. As the heart's rhythm becomes more coherent, it drives other oscillatory systems into entrainment to oscillate at the same frequency. Typically, entrainment is observed between heart rhythms, respiratory rhythms, and blood pressure oscillations; however, other biological oscillators, including very low frequency brain rhythms, craniosacral rhythms, and electrical potentials measured across the skin, can also become entrained.

Both the heart and the brain receive and respond to information about a future event 3 to 5 seconds before the event actually happens. Even more surprising is that the heart appears to receive this intuitive information approximately 1.5 seconds before the brain. Results of event-related potential and heartbeat evoked potential analyses suggest that the heart's afferent input to the brain may contain information pertaining to the future stimulus, thereby informing intuitive perception. During a state of psychophysiological coherence, the processing of prestimulus information in the brain is modified by the heart's afferent signals.

The heart also functions as a sensory organ, and is particularly sensitive and responsive to changes in a number of other psychophysiological systems. For example, heart rhythm patterns are continually modulated by changes in the activity of either branch of the ANS, and the heart's extensive intrinsic network of sensory neurons also enables it to detect and respond to variations in hormonal rhythms and patterns. In addition, the heart is itself an endocrine gland that manufactures and secretes multiple hormones and neurotransmitters. Cardiac afferent input not only exerts homeostatic effects via cardiovascular regulatory centers in the brain, but also influences the activity and function of higher brain centers involved in perceptual, cognitive, and emotional processing. The heart possesses a much more extensive communication system with the brain than do any of the body's other major organs. Through this system of afferent (ascending) neural pathways, the heart actually sends more information to the brain than the brain sends to the heart. The activation of positive emotions and the coherence mode leads to state in which higher cognitive faculties are facilitated [1].

Both neurophysiological and behavioral data indicate that afferent input from the heart and cardiovascular system influences the processing of sensory information. Cardiac related afferent input modulates a wide range of processes, including visual perception reaction times pain perception electrocortical activity and cognitive functions. The pattern of the heart's rhythmic activity and the corresponding pattern of cardiac afferent neural signals transmitted to the brain can inhibit or facilitate higher cognitive functions. During emotional stress, when the heart's rhythm is erratic or incoherent, this discordance communicates a signal to the brain that results in the inhibition of higher brain processes involved in perception, attention, reasoning, and creativity. This explains why we often cannot think clearly, make careless mistakes, and have little access to our creative capacities under stress. This "cortical inhibition" accounts that negative emotional states tend to produce more rigid and limited patterns of thought and action by reducing the ability to make accurate intuitive judgments [2].

There is a direct relationship between the heart rhythm patterns and the spectral information encoded in the frequency spectra of the magnetic field radiated by the heart. Thus, information about a person's emotional state is encoded in the heart's magnetic field which is communicated throughout the body and into the external environment. In essence, a bio-energetic communication system serves to inform function and behavior in highly coherent groups [3].

During positive emotional states, when the heart generates a harmonious, coherent pattern of activity, the resulting pattern of cardiac afferent input to the brain contributes to "cortical facilitation," whereby higher cognitive faculties are enhanced. This interaction between the heart and brain provides a physiological basis demonstrating a link between positive emotions and improved creativity, cognitive flexibility, innovative problem-solving, "flow," and intuition faculties that are also frequently enhanced during or following the generation of the psychophysiological coherence mode [4].

The state of psychophysiological coherence is marked by a number of notable internal changes. There is a quieting of the extraneous inner 
"noise" generated by the normal stream of thoughts and emotions, in conjunction with a positive emotion-driven global shift toward increased synchronization and harmony in psychophysiological processes. These changes not only enhance cognitive functioning, but also appear to result in increased receptivity and sensitivity to information that is normally outside the range of conscious awareness. The coherent structure of the electromagnetic field generated by the heart, enables the creation of an optimal communication channel between the wave fields generated by the body and by other objects and entities. Through an oscillatory resonance mechanism nonlocal information can be transmitted at hyper-speeds, by a quantum holographic process, having accurate foreknowledge of objects distant in space or events ahead in time, across macro and micro-scales of organization [5].

Human relationship influences our health and healing. In the clinical setting, divergent conceptual frameworks about healthcare can pose barriers to communication between patients and clinicians. Emerging concepts from quantum physics theory describe the energetic impact of human consciousness communication, and the mind-body connection. The current trend toward precision medicine is more personalized and holistic considering the patient as bio-psycho social being.

\section{References}

1. McCraty R, Deyhle A, Childre DL (2012) The global coherence initiative: Creating a coherent planetary standing wave. Global Advances in Health and Medicine: Improving Healthcare Outcomes Worldwide 1: 64-77

2. Fredrickson BL, Branigan C (2005) "Positive emotions broaden the scope of attention and thought-action repertoires." Cognition and Emotion 19: 313-332.

3. Morris S:M: (2010). Achieving Collective Coherence; Group Effects on Heart Rate Variability Coherence and Heart Rhythm Synchronization. Alternative Therapies in Health; Medicine, A peer reviewed journal, 2010; 16(4):62-72

4. Bolte A, Goschke T, Kuhkl J. (2003). Emotion and Intuition Effects of Positive and Negative Mood on Implicit Judgments of Semantic Coherence. Psychological Sci. 2003 Sep; 14(5):416-21

5. Bradley R:T (2006). The psychophysiology of entrepreneurial intuition: A quantum holographic theory. In proceedings of the Third AGSE International Entrepreneurship research exchange, February 8-10 2006. Auckland, New Zealand

Copyright: $\mathbb{C} 2020$ Mordeniz C. This is an open-access article distributed under the terms of the Creative Commons Attribution License, which permits unrestricted use, distribution, and reproduction in any medium, provided the original author and source are credited. 